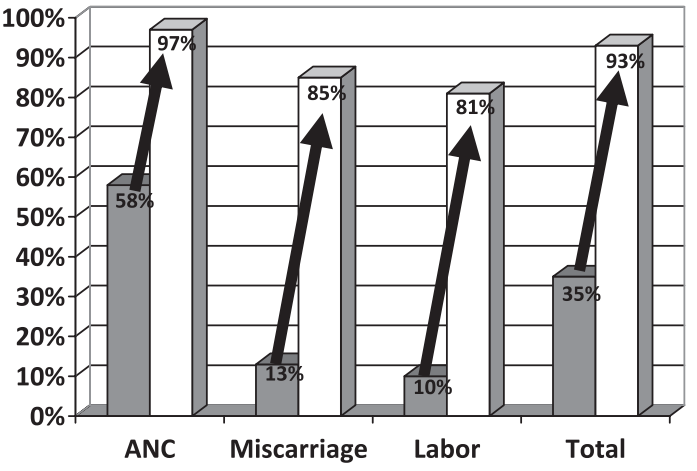

$\square 2009$ (RPR screening) $\square 2010$ (PRS screening)

Abstract P1-S6.35 Figure 1 Coverage of Syphilis screening by year and service Callao, Peru 2009-2010.

Conclusions Implementation of syphilis rapid test proved feasible, acceptable and effective in improving screening and treatment coverage and can serve as a powerful catalyst for improvements in quality of care.

\section{P1-S6.36 COST-EFFECTIVENESS OF RAPID TESTS TO IMPROVE ACCESS TO SYPHILIS PREVENTION AMIONG PREGNANT WOMEN IN PERU}

doi:10.1136/sextrans-2011-050108.260

${ }^{1} \mathrm{P}$ Mallma, ${ }^{1} \mathrm{C}$ Carcamo, ${ }^{1} \mathrm{M}$ Valderrama, ${ }^{1} \mathrm{M}$ Chiappe, ${ }^{1} \mathrm{~S}$ La Rosa, ${ }^{1} \mathrm{P}$ Garcia, ${ }^{2} \mathrm{M}$ Weaver, ${ }^{2} \mathrm{~K}$ Holmes, ${ }^{3} \mathrm{~F}$ Terris-Prestholt, ${ }^{3}$ Rossana Peeling. ${ }^{1}$ School of Public Health, Universidad Peruana Cayetano Heredia, Lima, Peru, ${ }^{2}$ University of Washington, Seattle, USA; ${ }^{3}$ London School of Hygiene and Tropical Medicine, London, UK

Background Several recent articles compared the cost and costeffectiveness syphilis testing strategies to avert congenital syphilis in settings with high syphilis prevalence. Current study contributes analysis in low-prevalence setting. Methods. Rapid syphilis testing (RST) was implemented at two different settings, both with syphilis prevalences of around 1\%: (a) The Ventanilla-Network of outpatient clinics and a small hospital at a peripheral district in Peru, where the rapid syphilis test was implemented together with the rapid HIV testing (One finger stick, two tests"); (b) The National Maternal and Perinatal Institute (INMP) a tertiary hospital with a high number of patients, and Initially in both settings the only test available was RPR with low coverage. For the costing of RPR we included supplies, capital costs, human resources and costs associated to treatment. For the costing of RST, we included also start up costs (advocacy meetings with authorities, training, supervision, monitoring) and the cost of implementing a quality assurance system. Cost-effectiveness analyses compared the cost of screening and treatment for the joint program to the disability adjusted lifeyears saved when congenital syphilis was averted.

Results For the Ventanilla-Network the total cost was $\$ 5.98$ for RST and $\$ 5.22$ for RPR per woman screened and $\$ 580.83$ and $\$ 1845.55$ respectively per woman treated. In contrast, the total cost was $\$ 2.53$ for rapid syphilis test and $\$ 3.15$ for RPR per woman screened (the lower costs probably associated to the economy of scale, due to the large number of women seen at the INMP) and $\$ 336.80$ and $\$ 1051.59$ at INMP. At Ventanilla-Network, the cost per DALY saved from averting cases of congenital syphilis was $\$ 35.23$ for rapid syphilis test and $\$ 111.95$ for RPR. In incremental analysis, the rapid test was cost-saving. At INMP, the cost per DALY saved was $\$ 20.43$ for rapid syphilis test and $\$ 63.79$. For the Ventanilla-Network and the INMP the RST was cost-effective by the WHO standard of \$64/ DALY.

Conclusion Syphilis screening is cost-effective even in a low-prevalence setting. To the extent that HIV rapid tests are funded by PMTCT programs, the cost of scaling up rapid syphilis tests would be lower than these estimates, because the joint cost of blood sample collection would be borne by the PMTCT program.

\section{P1-S6.37 CAN RAPID SYPHILIS TESTS BE IMPLEMENTED AND IMPROVE SCREENING FOR MATERNAL SYPHILIS IN A THIRD LEVEL HOSPITAL?}

doi:10.1136/sextrans-2011-050108.26

S La Rosa Roca, P Garcia. Cayetano Heredia University, Lima, Peru

Background Syphilis continues to be one of the most important causes of maternal and child morbi-mortality, frequently being more common than HIV. Ideal diagnostic test should provide rapid and accurate results to provide timely diagnosis and treatment. Rapid syphilis testing is an excellent option. The objective of this study is to determine the feasibility of the implementation of Rapid Syphilis Testing (RST, Syphilis 3.0 BioLine) and the impact on maternal screening for syphilis at a third level hospital in Peru: the National Maternal and Perinatal Institute (INMP).

Methods Between February and December of 2010, RST was implemented in the Antenatal care (ANC), labour and miscarriage services. The National guidelines recommended screening for syphilis in all those services with RPR. Health workers (midwives, nurses and laboratory technicians, depending on the service) were properly trained on the use of RST and their performance was monitored throughout the study.

Results RST was performed on 15116 women, with a prevalence of syphilis (RPR reactive, TPPA positive) of $1 \%$ for ANC, $0.7 \%$ for miscarriage services and $0.8 \%$ at labour. The coverage of screening for syphilis improved from $82 \%$ with RPR to $99 \%$ with RST at the ANC services; from $0 \%$ to $91 \%$ at the miscarriage services and from $79 \%$ to $94 \%$ at labour. At baseline, at the ANC visit, the results of the RPR were only available 15 days after the visit, resulting in a low coverage for treatment when the test was positive, 39\%. With the implementation of the RST, the results and treatment were given immediately at the same visit, resulting on treatment coverage of $95 \%$. The RST was very well accepted by the providers and patients.

Conclusions This study shows the feasibility, acceptability and improvements on screening associated to the implementation of RST in a third level hospital. In addition of improving coverage and treatment, our results triggered changes in the institutional policies for syphilis control.

\section{P1-S6.38 INTERPRETATION OF ROUTINE DATA FROM A YOUTH FRIENDLY CLINIC IN REGION F, JOHANNESBURG}

doi:10.1136/sextrans-2011-050108.262

S T Lalla-Edward. Wits Institute for Sexual and Reproductive Health, HIV and related diseases, Johannesburg, South Africa

Background The Wits Institute for Sexual and Reproductive Health, HIV and Related Diseases (WrHI)'s Youth Friendly Programme aims to engage with young people and make reproductive healthcare accessible and non-threatening. The team educates and informs young people about HIV and has specially sensitised nurses and 